\title{
Activities of the USP Project Team on Dissolution Calibration
}

\author{
Vivian Gray ${ }^{1}$, Mahendra Barot ${ }^{6}$, Pranab Bhattacharyya ${ }^{4}$, John Burmicz ${ }^{6}$, \\ Bryan Crist ${ }^{6}$, Thomas Foster ${ }^{1}$, Royal Hanson ${ }^{6}$, Herman Lam ${ }^{5}$, Lewis Leeson ${ }^{1}$, \\ John Mauger ${ }^{1}$, Terry Moore ${ }^{2}$, Werner Mueller6, Mary Oates ${ }^{3}$, Jean-Louis \\ Raton ${ }^{6}$, William Brown?
}

T he text of the USP general test chapter $<711>$ Dissolution requires the use of calibrator tablets as a part of the system suitability of a dissolution test apparatus. Only a suitable apparatus may be used in dissolution testing. Failures to meet the calibrator requirements not only put data obtained on the failing assembly in doubt but cost resources in failure investigation and may ultimately lead to the assembly being taken out of service. A high rate of failures perceived by users has been ascribed to the variability of the tablets. The entire dissolution community (USP staff and experts, industry and regulators) wants to ensure that dissolution calibration is a value-added activity. The USP Project Team on Dissolution Calibration was formed to initiate dialog and advance that concept.

The Project Team represents PhRMA, GPhA, FDA, CVG, and dissolution apparatus manufacturers. All are recognized as stakeholders in the USP dissolution calibrator program. The Council of Experts is represented on the Project Team by Thomas Foster, chair of the Biopharmaceutics Expert Committee, and by Vivian Gray, John Mauger, and Lewis Leeson, members of the Expert Committee. The Project team selected Vivian Gray as chair. Will Brown serves as USP staff manager for the team.

Since its first meeting on August 16,2001, the Project Team has met four times. Topics addressed by the Team include: the protocol of collaborative studies, possibility of a retest on calibration failure, enhanced mechanical calibration, and properties of an ideal calibrator tablet.

\section{Protocol of Collaborative Studies}

USP conducts a collaborative study to determine appropriate ranges for each lot of calibrator tablets. At their January 2003 meeting, the Team requested the opportunity to review the USP protocol for a planned Salicylic Acid Tablets collaborative study. A working group was identified and charged with giving comments on the existing protocol. The actions taken as a result of the working group's comments have allowed greater freedom by participants in several aspects of the collaborative study.

The requirement that the identical procedure for preparing standard solutions be followed to the letter was revised to allow larger or smaller masses and volumes to be used as long as the alternative preparations provide an equivalent accuracy. The working group recommended that the requirement to use standard solutions, freshly prepared, be modified to allow solutions previously prepared if stability of the solutions was demonstrated. This change was adopted, provided solutions were prepared by the analyst performing the dissolution so that an estimate of intermediate precision could be made. A final suggestion made by the working group and adopted in the revised protocol was to allow spectrophotometer cell path lengths other than $1 \mathrm{~cm}$ as originally specified.

\section{Possibility of Retest on Calibration Failure}

A continuing concern is what to do when a dissolution assembly fails calibration. The Project Team expressed interest in the possibility that some mechanism might be found so that the failing result could be verified by a retest. This issue is of interest in part because the acceptance ranges are calculated for individual vessel positions while a typical dissolution assembly consists of not less than six vessels. A failure to pass at one position results in the retest of all vessel positions and the possibility that the entire assembly might be taken out of service. Dr. Walter Hauck acting in his role as USP consulting statistician presented an analysis of the probability that a six-position assembly would pass calibration with the prednisone calibrator at the first trial by the various testing conditions based on the value of the mean of the data. The improvement in the probability of passing if a second trial was allowed was then estimated. The analysis of data from a collaborative study indicated that while retest might have some benefit for basket testing, it would offer minimal value for paddle testing. An overall change in the method for calculating the ranges for calibrators was instituted by USP based on the recognition of the dependence of the assembly passing on the results from each vessel. The recent increase in the ranges calculated for Prednisone Tablets, Lot O0C059, is an example of this new approach. [NOTE:The ranges for Prednisone Tablets, Lot O0C059, were changed effective December 6, 2004. The calibrator ranges can be seen at www.usp.org/standards/calibrators.html.]

\section{Enhanced Mechanical Calibration}

The Project Team has explored the possibility of replacing the current system suitability demonstration using the USP calibrator tablets with enhanced control of the various operational parameters. A proposed set of tightened tolerances was presented to the group for discussion. The USP general
${ }^{1}$ USP Biopharmaceutics Expert Committee

${ }^{2}$ Representing FDA

${ }^{3}$ Representing PhRMA

${ }^{4}$ Representing GPhA
${ }^{5}$ Representing CVG

${ }^{6}$ Representing apparatus manufacturers

'USP Staff Liaison, corresponding author 
chapter on dissolution gives measurements with tolerances that are viewed as not representing the current capability of dissolution apparatus manufacturers. A suggestion was made to propose that the USP adopt the tightened tolerances in its apparatus descriptions.

In the discussion several concerns were expressed. Not the least of the concerns was the concept that the USP standard is observed in more than 40 nations worldwide. The effect of such a change might have broad impact and has not been evaluated. The group decided to reconsider the suggestion at some later time pending further discussion and study.

\section{Properties of an Ideal Calibrator Tablet}

The first meeting of the Project Team produced a listing of the purposes of an ideal calibrator tablet. The list included demonstrating that the whole system works acceptably, providing an absolute standard useful for comparing apparatuses, capturing deviations, and a confirmation that the system works acceptably as a complement to mechanical calibration. A working group was formed to explore the properties of an ideal calibrator. The working group met separately and developed a list of attributes that were later presented to the entire Project Team. Due to the difficulty of measuring and understanding the effect on dissolution results, sensitivity to vibration was high on the list. The group recommended a list of desirable attributes among which stability, ease of manufacture and minimized toxicity were mentioned in addition to fulfilling the various purposes mentioned above.

In working group discussions, the difficulty of manufacturing an entirely new calibrator was recognized. Members of the working group suggested that the several products that have shown value should be evaluated. A challenge of the sensitivity of the performance of the several candidate products to changes in the dissolution system will be executed. The results will be combined with an evaluation of the other attributes to arrive at an estimation of the best candidate. The USP will pursue the manufacture of three tablet formulations, the current 10-mg prednisone tablets, a copy of the FDA NCDA \#2 tablet (10-mg prednisone), and a reproduction of the $50-\mathrm{mg}$ prednisone tablet that was distributed by USP as the disintegrating calibrator Lots $\mathrm{F}$ through L. The original 50-mg prednisone tablet was discontinued by USP as a result of the loss of the ability of the sponsor to manufacture it. However, through a cooperative agreement with the sponsor, the manufacturing information has been made available to USP for this project.

\section{Future}

This article has reported some of the accomplishments of the Project Team. More remains to be done. The design of the comparison experiments for the three tablet formulations is planned for mid-year 2005. An evaluation of tightened tolerances for dissolution apparatuses remains as an interest of the team and suggests a study of the value and appropriateness of such a change. Calibration of apparatuses not currently given in the USP general chapters such as for Apparatus 4, flow-through cell, is also being considered for future work.

The successes of the team have resulted from the open participation by members and reflect the shared interest in making the system suitability requirement in the USP dissolution general chapter a value-added activity.

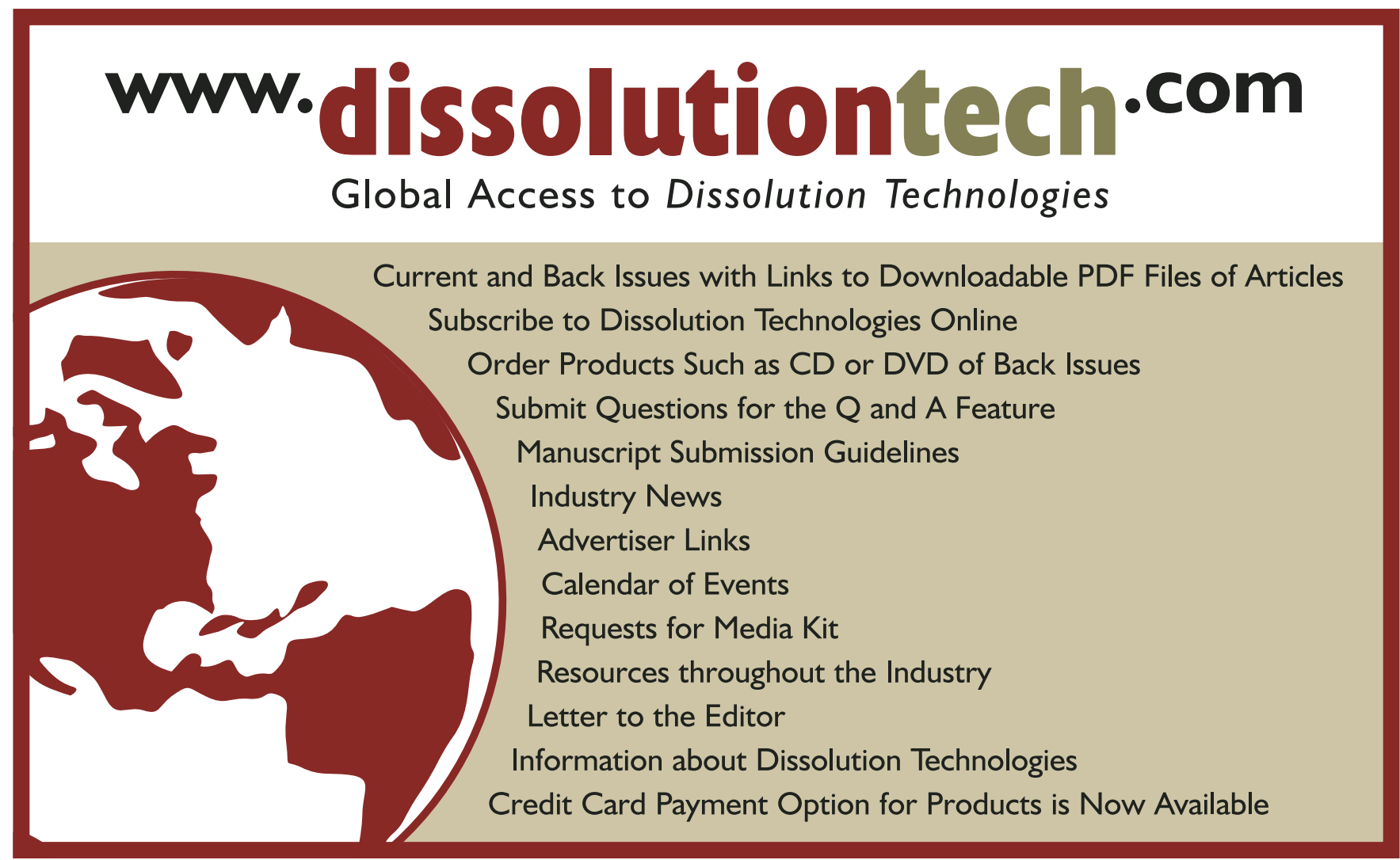

